\title{
Asymptotic Behavior of the Initial-boundary Value Problem of Landau-Lifshitz-Schrödinger Type
}

\author{
Yutian Lei
}

\begin{abstract}
This paper is concerned with the asymptotic behavior of the classical solutions of a Landau-Lifshitz-Schrödinger-type problem with initial-boundary values when the parameter $\varepsilon$ goes to zero. We establish several uniform estimates of $u_{\varepsilon}$ by a conservation result and the standard parabolic method. Based on these results, we obtain parabolic behavior in the dissipative case and non-parabolic behavior of the semi-classical limits of those solutions respectively.
\end{abstract}

\section{Introduction}

Let $G \subset \mathbb{R}^{2}$ be a bounded and simply connected domain with smooth boundary $\partial G$, $B=\left\{x \in \mathbb{R}^{2}|| x \mid<1\right\}, S^{2}=\left\{x=\left(x_{1}+i x_{2}, x_{3}\right) \in \mathbb{C} \times \mathbb{R}|| x \mid=x_{1}^{2}+x_{2}^{2}+x_{3}^{2}=1\right\}$, $S^{1}=\left\{x \in \mathbb{C} \times \mathbb{R} \mid x_{1}^{2}+x_{2}^{2}=1, x_{3}=0\right\}$. Sometimes we write the vector value function $u=\left(u_{1}+i u_{2}, u_{3}\right)=\left(u^{\prime}, u_{3}\right)$. Define $G_{T}=G \times(0, T]$ with $T \in(0, \infty)$. We are concerned with the limit behavior of the classical solution $u_{\varepsilon}: G_{T} \rightarrow S^{2}$ of the following problem of Landau-Lifshitz-Schrödinger type when the parameter $\varepsilon \rightarrow 0$

$$
\begin{cases}(a+i b) u_{t}^{\prime}=\Delta u^{\prime}+u^{\prime}|\nabla u|^{2}+\frac{1}{\varepsilon^{2}} u^{\prime} u_{3}^{2} & \text { on } G_{T}, \\ u_{3 t}=\Delta u_{3}+u_{3}|\nabla u|^{2}+\frac{1}{\varepsilon^{2}} u_{3}\left(u_{3}^{2}-1\right) & \text { on } G_{T}, \\ \left.u\right|_{\partial G \times \mathbb{R}^{+}}=g(x), & x \in G, \\ u(x, 0)=u_{0}(x), & \end{cases}
$$

where $a>0$ and $b$ are real constants, $g=\left(g^{\prime}, 0\right) \in C^{\infty}\left(\partial G, S^{1}\right), u_{0}=\left(u_{0}^{\prime}, 0\right) \in C^{\infty}\left(\bar{G}, S^{1}\right)$, $u_{0}(x)=g(x)$ as $x \in \partial G \times\{t=0\}$, and $\operatorname{deg}\left(g^{\prime}, \partial G\right)=0$.

Equation (1.1) is related to the study of the Schrödinger operator $\partial_{t}-(a+b i) \Delta$ (cf. $[7,17)$. When $a=1, b=0$ and $S^{2}$ is replaced by $\left\{x \in \mathbb{R}^{3}|| x \mid=1\right\}$, (1.1) can be rewritten as

$$
\begin{cases}u_{t}=\Delta u+u|\nabla u|^{2}+\frac{1}{\varepsilon^{2}}\left(u u_{3}^{2}-u_{3} e_{3}\right) & \text { on } G_{T}, \\ \left.u\right|_{\partial G \times \mathbb{R}^{+}}=g(x), & x \in G \\ u(x, 0)=u_{0}(x), & \end{cases}
$$

Received October 29, 2019; Accepted March 13, 2020.

Communicated by Jenn-Nan Wang.

2010 Mathematics Subject Classification. 35B40, 35K51, 35Q55.

Key words and phrases. Landau-Lifshitz-Schrödinger equation, uniform estimate, heat flow of harmonic map. 
where $e_{3}=(0,0,1)$. The system $(1.2)$ arises in the study of high-energy physics (cf. 10 , 16]). It controls the dynamics of planar ferromagnets and antiferromagnets. If the term $\frac{1}{\varepsilon^{2}}\left(u u_{3}^{2}-u_{3} e_{3}\right)$ is replaced by $\frac{1}{\varepsilon^{2}} u\left(1-|u|^{2}\right)$ and $S^{2}$ is replaced by $\mathbb{R}^{2},(1.2)$ becomes the Ginzburg-Landau system introduced in the theory of superconductors (see [2, 13, 18]). When $a=0$ and $b=1$, it is associated with the Gross-Pitaevskii-type equation (cf. 9,18 , 20]).

By virtue of $\operatorname{deg}\left(g^{\prime}, \partial G\right)=0$, there exists $\theta_{1}(x) \in C^{\infty}(\partial G)$ and $\theta_{2}(x) \in C^{\infty}(\bar{G})$ such that $g^{\prime}=e^{i \theta_{1}}$ on $\partial G$ and $u_{0}^{\prime}=e^{i \theta_{2}}$ in $G$, where $\left.\theta_{2}\right|_{\partial G}=\theta_{1}$. Clearly, the following problem

$$
\begin{cases}a \theta_{t}=\Delta \theta & \text { on } G_{T}, \\ \left.\theta\right|_{\partial G \times \mathbb{R}^{+}}=\theta_{1}, & \\ \theta(x, 0)=\theta_{2}(x), & x \in G\end{cases}
$$

has a unique solution $\theta(x, t)$. Set $u_{*}^{\prime}=e^{i \theta}$. Then $u_{*}^{\prime}$ is the unique solution to the problem (up to periods)

$$
\begin{cases}a w_{t}=\Delta w+w|\nabla w|^{2} & \text { on } G_{T}, \\ \left.w\right|_{\partial G \times \mathbb{R}^{+}}=g^{\prime}(x), & \\ w(x, 0)=u_{0}^{\prime}(x), & x \in G .\end{cases}
$$

It is a heat flow of harmonic map.

In Section 2, we will establish a conservation of energy. Based on this result, $u_{\varepsilon}$ converges to $\left(u_{*}^{\prime}, 0\right)$ when $\varepsilon \rightarrow 0$ as in [1,8], where $u_{*}^{\prime}$ is a heat flow of harmonic map. Thus, investigating the asymptotic behavior of the solution $u_{\varepsilon}$ of the Landau-Lifshitz-Schrödinger problem is helpful to well understand the properties of the heat flow of harmonic maps. On the contrary, we may also understand the asymptotic properties of $u_{\varepsilon}$ by means of the corresponding properties of $u_{*}^{\prime}$.

Theorem 1.1. Assume $u_{\varepsilon}: G_{T} \rightarrow S^{2}$ is a solution to 1.1), where $T<\infty$ is independent of $\varepsilon$. Then as $\varepsilon \rightarrow 0$,

$$
\begin{aligned}
u_{\varepsilon} & \rightarrow\left(u_{*}^{\prime}, 0\right) & & \text { weakly }{ }^{*} \text { in } L^{\infty}\left(0, T ; H^{1}\left(G, S^{2}\right)\right), \\
\frac{\partial}{\partial t} u_{\varepsilon} & \rightarrow \frac{\partial}{\partial t}\left(u_{*}^{\prime}, 0\right) & & \text { weakly in } L^{2}\left(0, T ; L^{2}\left(G, S^{2}\right)\right), \\
u_{\varepsilon} & \rightarrow\left(u_{*}^{\prime}, 0\right) & & \text { in } L^{2}\left(0, T ; L^{2}\left(G, S^{2}\right)\right), \\
u_{\varepsilon 3} & \rightarrow 0 & & \text { in } C^{\alpha, \alpha / 2}\left(\overline{G_{T}}\right) \text { for some } 0<\alpha<1 .
\end{aligned}
$$

Here, $u_{*}^{\prime}$ solves 1.3 .

The first three results can be deduced directly by using energy estimate. The last result relies on the estimate of $\left\|u_{\varepsilon 3}\right\|_{W_{p}^{2,1}\left(\overline{G_{T}}\right)}$. This type of estimate has already been obtained 
for evolution problems of the Ginzburg-Landau-type (cf. [6]) and the Landau-Lifshitztype (cf. [11]). These problems are distinctly different. The Landau-Lifshitz equation is more difficult to handle since it only satisfies the natural growth condition (with respect to $|\nabla u|^{2}$ ), unlike the Ginzburg-Landau equation satisfying the controllable growth condition. The computations have been developed in the context of the harmonic maps and can be generalized to included the anisotropic perturbation and the evolution in time (cf. [4 $5:$ : 15]). We will establish the analogous estimates for Landau-Lifshitz-Schrödinger problem (1.1) in Section 3 ,

Finally, in Section 4, we rescale $u_{\varepsilon}$ in time as in 3,14

$$
v_{\varepsilon}(x, t)=u_{\varepsilon}(x, \varepsilon t) .
$$

Then the function $v_{\varepsilon}=\left(v_{\varepsilon}^{\prime}, v_{\varepsilon 3}\right)$ satisfies

$$
\begin{cases}(a+i b) \frac{1}{\varepsilon} v_{t}^{\prime}=\Delta v^{\prime}+v^{\prime}|\nabla v|^{2}+\frac{1}{\varepsilon^{2}} v^{\prime} v_{3}^{2} & \text { on } G_{T}, \\ \frac{1}{\varepsilon} v_{3 t}=\Delta v_{3}+v_{3}|\nabla v|^{2}+\frac{1}{\varepsilon^{2}} v_{3}\left(v_{3}^{2}-1\right) & \text { on } G_{T}, \\ \left.v\right|_{\partial G \times \mathbb{R}^{+}}=g(x), & \\ v(x, 0)=u_{0}(x), & x \in G .\end{cases}
$$

Let $b \neq 0$. Clearly, the following hyperbolic problem

$$
\begin{cases}\theta_{t t}=\frac{2}{b^{2}} \Delta \theta & \text { on } G_{T}, \\ \left.\theta\right|_{\partial G \times \mathbb{R}^{+}}=\theta_{1}, & \\ \theta(x, 0)=\theta_{2}(x), & x \in G\end{cases}
$$

has a unique solution $\theta(x, t)$. Set $v_{*}^{\prime}=e^{i \theta}$, then $v_{*}^{\prime}$ is the unique solution to the problem (up to periods)

$$
\begin{cases}i w_{t}=-\frac{2 w}{b^{2}} \int_{0}^{t}[\operatorname{Im}(\bar{w} \Delta w)] d \tau & \text { on } G_{T}, \\ \left.w\right|_{\partial G \times \mathbb{R}^{+}}=g^{\prime}(x), & x \in G . \\ w(x, 0)=u_{0}^{\prime}(x), & \end{cases}
$$

The following result shows the limit relation between $v_{\varepsilon}$ and $\left(v_{*}^{\prime}, 0\right)$, and the nonparabolic behavior of $v_{*}^{\prime}$.

Theorem 1.2. Assume $v_{\varepsilon}: G_{T} \rightarrow S^{2}$ is a solution to (1.4), where $T<\infty$ is independent of $\varepsilon$. If $a \neq a^{2}+b^{2}$,

$$
\begin{aligned}
v_{\varepsilon} & \rightarrow\left(v_{*}^{\prime}, 0\right) & & \text { weakly }{ }^{*} \text { in } L^{\infty}\left(0, T ; H^{1}\left(G, S^{2}\right)\right), \\
\frac{\partial}{\partial t} v_{\varepsilon} & \rightarrow \frac{\partial}{\partial t}\left(v_{*}^{\prime}, 0\right) & & \text { weakly in } L^{2}\left(0, T ; L^{2}\left(G, S^{2}\right)\right), \\
v_{\varepsilon} & \rightarrow\left(v_{*}^{\prime}, 0\right) & & \text { in } L^{2}\left(0, T ; L^{2}\left(G, S^{2}\right)\right),
\end{aligned}
$$


when $\varepsilon \rightarrow 0$. Here, $v_{*}^{\prime}$ is a harmonic map on $G$ with the boundary value $g^{\prime}(x)$. On the other hand, when $a=0, v_{*}^{\prime}$ must solve (1.5) as long as the convergence results above still hold true.

\section{Parabolic behavior}

First we have a conservation result.

Proposition 2.1. Assume $u_{\varepsilon}: G \times \mathbb{R}^{+} \rightarrow S^{2}$ solves (1.1). Then we have

$$
\sup _{t>0}\left[\int_{0}^{t} \int_{G}\left(a\left|\frac{\partial}{\partial t} u_{\varepsilon}^{\prime}\right|^{2}+\left|\frac{\partial}{\partial t} u_{\varepsilon 3}\right|^{2}\right) d x d \tau+E_{\varepsilon}\left(u_{\varepsilon}(x, t)\right)\right]=\frac{1}{2}\left\|\nabla u_{0}\right\|_{2}^{2} .
$$

Here

$$
E_{\varepsilon}(u)=\frac{1}{2} \int_{G}|\nabla u|^{2} d x+\frac{1}{2 \varepsilon^{2}} \int_{G}\left|u_{3}\right|^{2} d x .
$$

Proof. Taking the real part and imaginary part of the first equation in $(1.1)$ and combining the second equation in (1.1), we have the following three equations

$$
\begin{gathered}
a u_{1 t}-b u_{2 t}=\Delta u_{1}+u_{1}|\nabla u|^{2}+\frac{1}{\varepsilon^{2}} u_{1} u_{3}^{2}, \\
b u_{1 t}+a u_{2 t}=\Delta u_{2}+u_{2}|\nabla u|^{2}+\frac{1}{\varepsilon^{2}} u_{2} u_{3}^{2}, \\
u_{3 t}=\Delta u_{3}+u_{3}|\nabla u|^{2}+\frac{1}{\varepsilon^{2}} u_{3}\left(u_{3}^{2}-1\right) .
\end{gathered}
$$

Multiply (2.2), 2.3) and (2.4) with $u_{1 t}, u_{2 t}$ and $u_{3 t}$, respectively. Integrating over $G$, we get

$$
\begin{aligned}
& a \int_{G}\left|u_{1 t}\right|^{2} d x-b \int_{G} u_{1 t} u_{2 t} d x \\
&= \int_{G} u_{1 t} \Delta u_{1} d x+\frac{1}{2} \int_{G}\left(\left|u_{1}\right|^{2}\right)_{t}|\nabla u|^{2} d x+\frac{1}{2 \varepsilon^{2}} \int_{G}\left(\left|u_{1}\right|^{2}\right)_{t} u_{3}^{2} d x \\
& b \int_{G} u_{1 t} u_{2 t} d x+a \int_{G}\left|u_{2 t}\right|^{2} d x \\
&= \int_{G} u_{2 t} \Delta u_{2} d x+\frac{1}{2} \int_{G}\left(\left|u_{2}\right|^{2}\right)_{t}|\nabla u|^{2} d x+\frac{1}{2 \varepsilon^{2}} \int_{G}\left(\left|u_{2}\right|^{2}\right)_{t} u_{3}^{2} d x \\
& \int_{G}\left|u_{3 t}\right|^{2} d x=\int_{G} u_{3 t} \Delta u_{3} d x+\frac{1}{2} \int_{G}\left(\left|u_{3}\right|^{2}\right)_{t}|\nabla u|^{2} d x+\frac{1}{2 \varepsilon^{2}} \int_{G}\left(\left|u_{3}\right|^{2}\right)_{t}\left(u_{3}^{2}-1\right) d x
\end{aligned}
$$

Noting $u_{t}=g_{t}=0$ on $\partial G$, we obtain by Green's theorem that

$$
\int_{G} u_{i t} \Delta u_{i} d x=-\frac{d}{2 d t} \int_{G}\left|\nabla u_{i}\right|^{2} d x, \quad i=1,2,3
$$


Insert into 2.5), (2.6) and (2.7), respectively. Noting $|u|=1$, we have $\left(|u|^{2}\right)_{t}=0$. Thus, combining the three results yields

$$
\int_{G} a\left(\left|u_{1 t}\right|^{2}+\left|u_{2 t}\right|^{2}\right) d x+\int_{G}\left|u_{3 t}\right|^{2} d x=-\frac{d}{d t} E_{\varepsilon}(u(x, t)) .
$$

Integrating from 0 to $t$, we deduce that, for all $t>0$,

$$
\int_{0}^{t} \int_{G}\left(a\left|\frac{\partial}{\partial t} u_{\varepsilon}^{\prime}\right|^{2}+\left|\frac{\partial}{\partial t} u_{\varepsilon 3}\right|^{2}\right) d x d \tau+E_{\varepsilon}\left(u_{\varepsilon}(x, t)\right)=E_{\varepsilon}(u(x, 0))=\frac{1}{2}\left\|\nabla u_{0}\right\|_{2}^{2} .
$$

Proposition 2.1 is proved.

By the conservation result, we can see the parabolic behavior of $u_{\varepsilon}$ when $\varepsilon \rightarrow 0$.

Proposition 2.2. Assume $u_{\varepsilon}: G_{T} \rightarrow S^{2}$ is a solution to 1.1), where $T<\infty$. Then as $\varepsilon \rightarrow 0$,

$$
\begin{aligned}
u_{\varepsilon} & \rightarrow\left(u_{*}^{\prime}, 0\right) & & \text { weakly }{ }^{*} \text { in } L^{\infty}\left(0, T ; H^{1}\left(G, S^{2}\right)\right), \\
\frac{\partial}{\partial t} u_{\varepsilon} & \rightarrow \frac{\partial}{\partial t}\left(u_{*}^{\prime}, 0\right) & & \text { weakly in } L^{2}\left(0, T ; L^{2}\left(G, S^{2}\right)\right), \\
u_{\varepsilon} & \rightarrow\left(u_{*}^{\prime}, 0\right) & & \text { in } L^{2}\left(0, T ; L^{2}\left(G, S^{2}\right)\right) .
\end{aligned}
$$

Here, $u_{*}^{\prime}$ solves 1.3 .

Proof. By Proposition 2.1, we can find a subsequence $\varepsilon_{k}$ such that as $\varepsilon_{k} \rightarrow 0$,

$$
\begin{cases}u_{\varepsilon_{k}} \rightarrow w & \text { weakly* in } L^{\infty}\left(0, T ; H^{1}\left(G, S^{2}\right)\right) \\ \frac{\partial}{\partial t} u_{\varepsilon_{k}} \rightarrow \frac{\partial}{\partial t} w & \text { weakly in } L^{2}\left(0, T ; L^{2}\left(G, S^{2}\right)\right) \\ u_{\varepsilon_{k}} \rightarrow w & \text { in } L^{2}\left(0, T ; L^{2}\left(G, S^{2}\right)\right) .\end{cases}
$$

We claim that $w=\left(u_{*}^{\prime}, 0\right)$, where $u_{*}^{\prime}$ is the unique solution to $(1.3)$. When $\varepsilon \rightarrow 0$, by (2.1) we know $\sup _{t>0} \int_{G} u_{\varepsilon_{k} 3}^{2} d x \rightarrow 0$. This result shows $w_{3}=0$ a.e. on $G_{T}$. Since $u_{\varepsilon_{k}}=u=\left(u^{\prime}, u_{3}\right)$ solves 1.1 , for all $\phi \in C_{0}^{\infty}\left(G_{T}\right)$, we have

$$
\begin{aligned}
\int_{G_{T}}\left(a u_{1 t}-b u_{2 t}\right) \phi d x d t & =\int_{G_{T}} \Delta u_{1} \phi d x d t+\int_{G_{T}} u_{1}|\nabla u|^{2} \phi d x d t+\frac{1}{\varepsilon^{2}} \int_{G_{T}} u_{1} u_{3}^{2} \phi d x d t \\
\int_{G_{T}}\left(b u_{1 t}+a u_{2 t}\right) \phi d x d t & =\int_{G_{T}} \Delta u_{2} \phi d x d t+\int_{G_{T}} u_{2}|\nabla u|^{2} \phi d x d t+\frac{1}{\varepsilon^{2}} \int_{G_{T}} u_{2} u_{3}^{2} \phi d x d t .
\end{aligned}
$$

Take $\phi=u_{2} \zeta$ and $\phi=u_{1} \zeta$ in the first and second integral equations respectively. Here 
$\zeta \in C_{0}^{\infty}\left(G_{T}\right)$. Then,

$$
\begin{aligned}
& \int_{G_{T}}\left(a u_{1}-b u_{2}\right)\left(u_{2} \zeta\right)_{t} d x d t \\
= & \int_{G_{T}} \nabla u_{1} \nabla\left(u_{2} \zeta\right) d x d t-\int_{G_{T}} u_{1}|\nabla u|^{2} u_{2} \zeta d x d t-\frac{1}{\varepsilon^{2}} \int_{G_{T}} u_{1} u_{3}^{2} u_{2} \zeta d x d t, \\
& \int_{G_{T}}\left(b u_{1}+a u_{2}\right)\left(u_{1} \zeta\right)_{t} d x d t \\
= & \int_{G_{T}} \nabla u_{2} \nabla\left(u_{1} \zeta\right) d x d t-\int_{G_{T}} u_{2}|\nabla u|^{2} u_{1} \zeta d x d t-\frac{1}{\varepsilon^{2}} \int_{G_{T}} u_{2} u_{3}^{2} u_{1} \zeta d x d t .
\end{aligned}
$$

Subtracting one from the other yields

$$
\begin{aligned}
& \int_{G_{T}} a \zeta\left(u_{1} u_{2 t}-u_{2} u_{1 t}\right) d x d t-\int_{G_{T}} b \zeta\left(u_{1} u_{1 t}+u_{2} u_{2 t}\right) d x d t-\int_{G_{T}} b \zeta_{t}\left(u_{1}^{2}+u_{2}^{2}\right) d x d t \\
= & \int_{G_{T}} \nabla \zeta\left(u_{2} \nabla u_{1}-u_{1} \nabla u_{2}\right) d x d t .
\end{aligned}
$$

Letting $\varepsilon \rightarrow 0$ and using (2.8), we obtain

$$
a \int_{G_{T}} \zeta\left(w^{\prime} \wedge w_{t}^{\prime}\right) d x d t=\int_{G_{T}} \nabla \zeta\left(\nabla w^{\prime} \wedge w^{\prime}\right) d x d t .
$$

Integrating by parts, we get that for all $\zeta \in C_{0}^{\infty}\left(G_{T}\right)$,

$$
a \int_{G_{T}} \zeta\left(w^{\prime} \wedge w_{t}^{\prime}\right) d x d t=\int_{G_{T}} \zeta \operatorname{div}\left(w^{\prime} \wedge \nabla w^{\prime}\right) d x d t .
$$

Let $w^{\prime}=e^{i \theta}$, then $w_{t}^{\prime}=i e^{i \theta} \theta_{t}, \nabla w^{\prime}=i e^{i \theta} \nabla \theta$, and

$$
a\left(w^{\prime} \wedge w_{t}^{\prime}\right)=a \theta_{t}, \quad \operatorname{div}\left(w^{\prime} \wedge \Delta w^{\prime}\right)=\Delta \theta .
$$

Thus, 2.9 leads to

$$
a \theta_{t}=\Delta \theta \text {. }
$$

Since the limit $\left(u_{*}^{\prime}, 0\right)$ is unique, the convergence above can be generalized to all $\varepsilon$ instead of the subsequence $\varepsilon_{k}$. This implies that $u_{*}^{\prime}$ satisfies (1.3). Proposition 2.2 is proved.

Next, we will show that $\left|u_{\varepsilon}^{\prime}\right|$ is positive for sufficiently small $\varepsilon$. We first need a Lipschitz continuity result which can be deduced by (2.1). In fact, this is predictable because the $W^{1, \infty}$-function is Lipschitz continuous (cf. Exercise 8 of Chapter 6 in [12]).

Proposition 2.3. Assume $u=u_{\varepsilon}: \overline{G_{T}} \rightarrow S^{2}$ is a solution to (1.1). Then there exists a constant $C>0$ independent of $\varepsilon$ such that for any $x_{1}, x_{2} \in G$ and $t \in[0, T]$,

$$
\left|u\left(x_{1}, t\right)-u\left(x_{2}, t\right)\right| \leq C\left|x_{1}-x_{2}\right| .
$$


Proof. We give a complementary definition of $u \equiv 0$ on $\mathbb{R}^{2} \backslash \overline{G_{T}}$. Thus, $u$ is defined on $\mathbb{R}^{2}$.

We first consider inner estimate. Let $J_{\eta}$ be the mollification operator, and write

$$
u_{\eta}(x, t)=J_{\eta} u(x, t)=\int_{0}^{T} \int_{\mathbb{R}^{2}} j_{\eta}(x-y, t-\tau) u(y, \tau) d y d \tau
$$

where $0<\eta<t<T-\eta$. Denote $u_{\varepsilon}$ by $u$. For any $x_{1}, x_{2} \in G^{\lambda}:=\{x \in G \mid \operatorname{dist}(x, \partial G) \geq$ $\lambda\}$, there holds

$$
\begin{aligned}
& u_{\eta}\left(x_{1}, t\right)-u_{\eta}\left(x_{2}, t\right) \\
= & \int_{0}^{T} \int_{\mathbb{R}^{2}} \int_{0}^{1} \frac{d}{d s} j_{\eta}\left(s x_{1}+(1-s) x_{2}-y, t-\tau\right) u(y, \tau) d s d y d \tau \\
= & \left(x_{1}-x_{2}\right) \int_{0}^{T} \int_{\mathbb{R}^{2}} \int_{0}^{1} \nabla_{x} j_{\eta}\left(s x_{1}+(1-s) x_{2}-y, t-\tau\right) u(y, \tau) d s d y d \tau \\
= & -\left(x_{1}-x_{2}\right) \int_{0}^{1} \int_{\mathbb{R}^{2}} \int_{0}^{T} \nabla_{y} j_{\eta}\left(s x_{1}+(1-s) x_{2}-y, t-\tau\right) u(y, \tau) d \tau d y d s \\
= & \left(x_{1}-x_{2}\right) \int_{0}^{1} \int_{\mathbb{R}^{2}} \int_{0}^{T} j_{\eta}\left(s x_{1}+(1-s) x_{2}-y, t-\tau\right) \nabla_{y} u(y, \tau) d \tau d y d s .
\end{aligned}
$$

Applying (2.1) we obtain

$$
\begin{aligned}
& \left|u_{\eta}\left(x_{1}, t\right)-u_{\eta}\left(x_{2}, t\right)\right| \\
\leq & \left|x_{1}-x_{2}\right| \int_{0}^{1} \int_{\mathbb{R}^{2}} \int_{0}^{T}\left|j_{\eta}\left(s x_{1}+(1-s) x_{2}-y, t-\tau\right)\right|\left|\nabla_{y} u(y, \tau)\right| d \tau d y d s \\
\leq & C\left|x_{1}-x_{2}\right| .
\end{aligned}
$$

Here $C>0$ is independent of $\eta$ and $\varepsilon$. Letting $\eta \rightarrow 0$, we can derive 2.10 for $x_{1}, x_{2} \in G^{\lambda}$.

Next, we give the estimate near the boundary. Let $x_{0} \in \partial G$. Without loss of generality, we assume $G \cap B_{2 R}\left(x_{0}\right)=\left\{\left(x^{1}, x^{2}\right) \mid x^{2}>0\right\} \cap B_{2 R}\left(x_{0}\right)$. Let $J_{\eta}^{+}$be the mollification operator

$$
u_{\eta}^{+}(x, t)=J_{\eta}^{+} u(x, t)=\int_{0}^{T} \int_{\mathbb{R}^{2}} j_{\eta}\left(x^{1}-y^{1}\right) j_{\eta}\left(x^{2}-y^{2}+2 \varepsilon\right) j_{\eta}(t-\tau) u(y, \tau) d y d \tau
$$

where $0<t \leq T$. For any $x_{1}=\left(x_{1}^{1}, x_{1}^{2}\right), x_{2}=\left(x_{2}^{1}, x_{2}^{2}\right) \in G \cap B_{2 R}\left(x_{0}\right)$, using the same argument above, we can also deduce 2.10 near the boundary $\partial G$. Similarly, we also get 2.10 near $t=0$. Proposition 2.3 is complete.

Proposition 2.4. Assume $u_{\varepsilon}: \overline{G_{T}} \rightarrow S^{2}$ is a solution to 1.1 . Then $\left|u_{\varepsilon}^{\prime}\right| \geq 1 / 2$ in $\overline{G_{T}}$ as long as $\varepsilon$ is sufficiently small.

Proof. We will prove that for each given $t \in(0, T]$,

$$
\left|u_{\varepsilon}^{\prime}(x, t)\right| \geq \frac{1}{2}, \quad \forall x \in G
$$


as long as $\varepsilon$ is sufficiently small. Otherwise, for some fixed $t_{0} \in(0, T]$, we can find $x_{\varepsilon} \in G$ satisfying

$$
\left|u_{\varepsilon}^{\prime}\left(x_{\varepsilon}, t_{0}\right)\right|<\frac{1}{2}
$$

According to Proposition 2.3, we have

$$
\begin{aligned}
\left|u_{\varepsilon}^{\prime}\left(x, t_{0}\right)\right| & \leq\left|u_{\varepsilon}^{\prime}\left(x, t_{0}\right)-u_{\varepsilon}^{\prime}\left(x_{\varepsilon}, t_{0}\right)\right|+\left|u_{\varepsilon}^{\prime}\left(x_{\varepsilon}, t_{0}\right)\right| \\
& \leq C\left|x-x_{\varepsilon}\right|+\frac{1}{2}<\frac{3}{4} \text { for }\left|x-x_{\varepsilon}\right|<\frac{1}{4 C} .
\end{aligned}
$$

On the other hand, 2.10 implies $\left|u_{\varepsilon}^{\prime}\left(x, t_{0}\right)\right|_{C^{0+1}(\bar{G})} \leq C$. By means of the compact embedding theorem, we have

$$
u_{\varepsilon}^{\prime}\left(x, t_{0}\right) \rightarrow u_{*}^{\prime}\left(x, t_{0}\right) \quad \text { in } C^{\alpha}(\bar{G}) \quad \text { as } \varepsilon \rightarrow 0,
$$

where $\alpha \in(0,1)$. Proposition 2.2 shows that the limit $u_{*}^{\prime}$ satisfies $\left|u_{*}^{\prime}\left(x, t_{0}\right)\right|=1$. This contradicts (2.12). Thus (2.11) holds true for any $t \in(0, T]$. In view of the initial-boundary condition, the proof of Proposition 2.4 is completed.

\section{Uniform estimates}

The main result of this section is the Hölder convergence of $u_{\varepsilon 3}$ when $\varepsilon \rightarrow 0$. First, we establish a uniform estimate on the boundary.

Proposition 3.1. Assume that $u_{\varepsilon}: G_{T} \rightarrow S^{2}$ is a solution to (1.1). Then, there exists a constant $C>0$ which is independent of $\varepsilon$ such that

$$
\int_{0}^{T} \int_{\partial G}\left|\frac{\partial u_{\varepsilon}}{\partial \nu}\right|^{2} d s d t \leq C
$$

where $\nu$ is the unit outward normal vector on $\partial G$.

Proof. Let $\mathbf{n} \in C^{\infty}\left(G, \partial B_{1}(0)\right)$ such that $\mathbf{n}=\nu$ on $\partial G$, where $\nu$ is the unit outward normal vector. Denote $u_{\varepsilon}$ by $u$. Multiply (2.2), (2.3) and (2.4) with $\mathbf{n} \cdot \nabla u_{1}, \mathbf{n} \cdot \nabla u_{2}$ and $\mathbf{n} \cdot \nabla u_{3}$ respectively and integrating on $G_{T}$. Since

$$
\int_{G} \Delta u_{i}\left(\mathbf{n} \cdot \nabla u_{i}\right) d x=\int_{\partial G}\left|\frac{\partial u_{i}}{\partial \nu}\right|^{2} d s-\int_{G} \nabla u_{i} \cdot \nabla\left(\mathbf{n} \cdot \nabla u_{i}\right) d x, \quad i=1,2,3
$$


we have

$$
\begin{aligned}
\int_{0}^{T} \int_{\partial G}\left|\frac{\partial u}{\partial \nu}\right|^{2} d s d t= & \int_{G_{T}} a\left(u_{t}^{\prime} \cdot\left(\mathbf{n} \cdot \nabla u^{\prime}\right)\right) d x d t+\int_{G_{T}} b \operatorname{det}\left(u_{t}^{\prime}\left(\mathbf{n} \cdot \nabla u^{\prime}\right)\right) d x d t \\
& +\int_{G_{T}} u_{3 t}\left(\mathbf{n} \cdot \nabla u_{3}\right) d x d t+\sum_{l=1}^{3} \int_{G_{T}} \nabla u_{l} \cdot \nabla\left(\mathbf{n} \cdot \nabla u_{l}\right) d x d t \\
& -\int_{G_{T}}\left(|\nabla u|^{2}(u \cdot(\mathbf{n} \cdot \nabla u))\right) d x d t-\frac{1}{\varepsilon^{2}} \int_{G_{T}} u_{3}^{2}(u \cdot(\mathbf{n} \cdot \nabla u)) d x d t \\
& +\frac{1}{\varepsilon^{2}} \int_{G_{T}} u_{3}\left(\mathbf{n} \cdot \nabla u_{3}\right) d x d t .
\end{aligned}
$$

Here $\operatorname{det}\left(u^{\prime}, v^{\prime}\right)=u_{1} v_{2}-u_{2} v_{1}$. Noting the smoothness of $\mathbf{n}$, from (2.1) and the Cauchy inequality, we can deduce

$$
\int_{G_{T}}\left[\left|a u_{1 t}+b u_{2 t}\right|\left|\mathbf{n} \cdot \nabla u_{1}\right|+\left|a u_{2 t}+b u_{1 t}\right|\left|\mathbf{n} \cdot \nabla u_{2}\right|+\left|u_{3 t}\right|\left|\mathbf{n} \cdot \nabla u_{3}\right|\right] d x d t \leq C .
$$

In addition, using (2.1) we also have

$$
\begin{aligned}
\sum_{l=1}^{3} \int_{G} \nabla u_{l} \cdot \nabla\left(\mathbf{n} \cdot \nabla u_{l}\right) d x & \leq C \int_{G}|\nabla u|^{2} d x+\frac{1}{2}\left|\int_{G} \mathbf{n} \cdot \nabla\left(|\nabla u|^{2}\right) d x\right| \\
& \leq C+\frac{1}{2} \int_{\partial G}|\nabla u|^{2} d s .
\end{aligned}
$$

Noting $|u|=1$, we get $\frac{1}{2} \nabla\left(|u|^{2}\right)=0$. Therefore,

$$
-\int_{G}|\nabla u|^{2}(u \cdot(\mathbf{n} \cdot \nabla u)) d x=-\frac{1}{2} \int_{G}|\nabla u|^{2}\left(\mathbf{n} \cdot \nabla\left(|u|^{2}\right)\right) d x=0 .
$$

In view of $u_{3}=0$ on $\partial G$, using 2.1 we can obtain that

$$
\begin{aligned}
& -\frac{1}{\varepsilon^{2}} \int_{G} u_{3}^{2}(u \cdot(\mathbf{n} \cdot \nabla u)) d x+\frac{1}{\varepsilon^{2}} \int_{G} u_{3}\left(\mathbf{n} \cdot \nabla u_{3}\right) d x \\
= & -\frac{1}{2 \varepsilon^{2}} \int_{G} u_{3}^{2}\left(\mathbf{n} \cdot \nabla\left(|u|^{2}\right)\right) d x+\frac{1}{2 \varepsilon^{2}} \int_{G}\left(\mathbf{n} \cdot \nabla u_{3}^{2}\right) d x \\
= & \frac{1}{2 \varepsilon^{2}} \int_{\partial G} u_{3}^{2} d s-\frac{1}{2 \varepsilon^{2}} \int_{G} u_{3}^{2}(\operatorname{div} \mathbf{n}) d x \leq C .
\end{aligned}
$$

Thus, by the boundary value condition in 1.1 , we get

$$
\int_{0}^{T} \int_{\partial G}\left|\frac{\partial u}{\partial \nu}\right|^{2} d s d t \leq C+\frac{1}{2} \int_{0}^{T} \int_{\partial G}\left|\frac{\partial g}{\partial \tau}\right|^{2} d s d t \leq C,
$$

where $\tau$ is the unit tangent vector on $\partial G$. Here $C>0$ is independent of $\varepsilon$. Proposition 3.1 is proved. 
Next, we will establish the uniform $W_{2}^{2,1}$-estimate of $u_{\varepsilon}$. Noting (2.1), we need the following result.

Proposition 3.2. Assume that $u_{\varepsilon}: G_{T} \rightarrow S^{2}$ is a solution to 1 .1. . Let $\left(x_{0}, t_{0}\right) \in \overline{G_{T}}$, $Q_{R}=\overline{G_{T}} \cap\left(B_{R}\left(x_{0}\right) \times\left[t_{0}, t_{0}+R^{2}\right]\right)$. Then, for suitably small $R>0$, there exists a constant $C=C(R, T)>0$ which is independent of $\varepsilon$, such that

$$
\left\|D^{2} u_{\varepsilon}\right\|_{L^{2}\left(Q_{R}\right)} \leq C \text {. }
$$

Proof. Differentiate (2.2), (2.3), and (2.4) with respect to $x_{j}$, then

$$
\begin{gathered}
a u_{1 x_{j} t}-b u_{2 x_{j} t}=u_{1 x_{i} x_{i} x_{j}}+\left(u_{1}|\nabla u|^{2}\right)_{x_{j}}+\frac{1}{\varepsilon^{2}}\left(u_{1} u_{3}^{2}\right)_{x_{j}}, \\
b u_{1 x_{j} t}+a u_{2 x_{j} t}=u_{2 x_{i} x_{i} x_{j}}+\left(u_{2}|\nabla u|^{2}\right)_{x_{j}}+\frac{1}{\varepsilon^{2}}\left(u_{2} u_{3}^{2}\right)_{x_{j}}, \\
u_{3 x_{j} t}=u_{3 x_{i} x_{i} x_{j}}+\left(u_{3}|\nabla u|^{2}\right)_{x_{j}}-\frac{1}{\varepsilon^{2}}\left(u_{3}\left|u^{\prime}\right|^{2}\right)_{x_{j}} .
\end{gathered}
$$

First we give the inner estimate. Let $\zeta=\zeta(x) \in C_{0}^{\infty}\left(\overline{B_{2 R\left(x_{0}\right)}},[0,1]\right)$ satisfy $\zeta=1$ in $B_{R}$, $|\nabla \zeta| \leq C R^{-1}$. Multiply (3.3), (3.4) and (3.5) by $\zeta^{2} u_{1 x_{j}}, \zeta^{2} u_{2 x_{j}}$ and $\zeta^{2} u_{3 x_{j}}$, respectively. Then integrating over $Q_{2 R}$, we get

$$
\begin{aligned}
& \frac{a}{2} \int_{Q_{2 R}} \zeta^{2}\left(\left|u_{1 x_{j}}\right|^{2}\right)_{t} d x d t-b \int_{Q_{2 R}} \zeta^{2} u_{2 x_{j} t} u_{1 x_{j}} d x d t \\
= & \int_{Q_{2 R}} \zeta^{2} u_{1 x_{i} x_{i} x_{j}} u_{1 x_{j}} d x d t+\int_{Q_{2 R}} \zeta^{2}\left(u_{1}|\nabla u|^{2}\right)_{x_{j}} u_{1 x_{j}} d x d t+\int_{Q_{2 R}} \frac{\zeta^{2}}{\varepsilon^{2}}\left(u_{1} u_{3}^{2}\right)_{x_{j}} u_{1 x_{j}} d x d t \\
& b \int_{Q_{2 R}} \zeta^{2} u_{1 x_{j} t} u_{2 x_{j}} d x d t+\frac{a}{2} \int_{Q_{2 R}} \zeta^{2}\left(\left|u_{2 x_{j}}\right|^{2}\right)_{t} d x d t \\
= & \int_{Q_{2 R}} \zeta^{2} u_{2 x_{i} x_{i} x_{j}} u_{2 x_{j}} d x d t+\int_{Q_{2 R}} \zeta^{2}\left(u_{2}|\nabla u|^{2}\right)_{x_{j}} u_{2 x_{j}} d x d t+\int_{Q_{2 R}} \frac{\zeta^{2}}{\varepsilon^{2}}\left(u_{2} u_{3}^{2}\right)_{x_{j}} u_{2 x_{j}} d x d t
\end{aligned}
$$

and

$$
\begin{aligned}
\frac{1}{2} \int_{Q_{2 R}} \zeta^{2}\left(\mid u_{\left.3 x_{j}\right|^{2}}\right)_{t} d x d t= & \int_{Q_{2 R}} \zeta^{2} u_{3 x_{i} x_{i} x_{j}} u_{3 x_{j}} d x d t+\int_{Q_{2 R}} \zeta^{2}\left(u_{3}|\nabla u|^{2}\right)_{x_{j}} u_{3 x_{j}} d x d t \\
& -\int_{Q_{2 R}} \frac{\zeta^{2}}{\varepsilon^{2}}\left(u_{3}\left|u^{\prime}\right|^{2}\right)_{x_{j}} u_{3 x_{j}} d x d t .
\end{aligned}
$$

Therefore, using Green's theorem and noting $\left(|u|^{2}\right)_{x_{j}}=0$, we obtain

$$
\begin{aligned}
& \quad \frac{a}{2} \int_{B_{2 R}} \zeta^{2}\left|u_{x_{j}}^{\prime}\right|^{2}\left(x, t_{0}+4 R^{2}\right) d x+\frac{1}{2} \int_{B_{2 R}} \zeta^{2}\left|u_{3 x_{j}}\right|^{2}\left(x, t_{0}+4 R^{2}\right) d x \\
& \quad+\int_{Q_{2 R}} \zeta^{2}\left|u_{x_{i} x_{j}}\right|^{2} d x d t+\frac{1}{\varepsilon^{2}} \int_{Q_{2 R}} \zeta^{2}\left|u_{3 x_{j}}\right|^{2} d x d t \\
& =\frac{a}{2} \int_{B_{2 R}} \zeta^{2}\left|u_{x_{j}}^{\prime}\right|^{2}\left(x, t_{0}\right) d x+\frac{1}{2} \int_{B_{2 R}} \zeta^{2}\left|u_{3 x_{j}}\right|^{2}\left(x, t_{0}\right) d x
\end{aligned}
$$




$$
\begin{aligned}
& +b \int_{Q_{2 R}} \zeta^{2} \operatorname{det}\left(u_{t}^{\prime}, u_{x_{j} x_{j}}^{\prime}\right) d x d t+2 b \int_{Q_{2 R}} \zeta \zeta_{x_{j}} \operatorname{det}\left(u_{t}^{\prime}, u_{x_{j}}^{\prime}\right) d x d t \\
& -2 \int_{Q_{2 R}} \zeta \zeta_{x_{j}}\left(u_{x_{j}} \cdot u_{x_{i} x_{j}}\right) d x d t+\int_{Q_{2 R}} \zeta^{2}|\nabla u|^{2}\left|u_{x_{j}}\right|^{2} d x d t \\
& +\frac{1}{\varepsilon^{2}} \int_{Q_{2 R}} \zeta^{2} u_{3}^{2}\left|u_{x_{j}}\right|^{2} d x d t .
\end{aligned}
$$

Here $B_{2 R}=\bar{G} \cap B_{2 R}\left(x_{0}\right)$. Using Cauchy's inequality and (2.1) to estimate the right-hand side of (3.6), for any $\delta \in(0,1)$, we obtain

$$
\begin{aligned}
\left|\int_{Q_{2 R}} \zeta^{2} \operatorname{det}\left(u_{t}^{\prime}, u_{x_{j} x_{j}}^{\prime}\right) d x d t\right| & \leq \delta \int_{Q_{2 R}} \zeta^{2}\left|u_{x_{j} x_{j}}\right|^{2} d x d t+C_{\delta} \int_{Q_{2 R}} \zeta^{2}\left|u_{t}^{\prime}\right|^{2} d x d t, \\
\left|\int_{Q_{2 R}} \zeta \zeta_{x_{j}} \operatorname{det}\left(u_{t}^{\prime}, u_{x_{j}}^{\prime}\right) d x d t\right| & \leq \delta \int_{Q_{2 R}} \zeta^{2}|\nabla u|^{2} d x d t+C_{\delta} \int_{Q_{2 R}}|\nabla \zeta|^{2}\left|u_{t}^{\prime}\right|^{2} d x d t, \\
\left|\int_{Q_{2 R}} \zeta \zeta_{x_{j}}\left(u_{x_{j}} \cdot u_{x_{i} x_{j}}\right) d x d t\right| & \leq \delta \int_{Q_{2 R}} \zeta^{2}\left|u_{x_{j} x_{j}}\right|^{2} d x d t+C_{\delta} \int_{Q_{2 R}}|\nabla \zeta|^{2}|\nabla u|^{2} d x d t, \\
\left.\left|\int_{Q_{2 R}} \zeta^{2}\right| \nabla u\right|^{2}\left|u_{x_{j}}\right|^{2} d x d t \mid & \leq C \int_{Q_{2 R}} \zeta^{2}|\nabla u|^{4} d x d t .
\end{aligned}
$$

To estimate the last term, we use the first equation of (1.1) and Proposition 2.4 to deduce that

$$
\frac{1}{\varepsilon^{2}} u_{3}^{2}=\frac{1}{\left|u^{\prime}\right|^{2}}\left|\bar{u}^{\prime}\left[(a+b i) u_{t}^{\prime}-\Delta u^{\prime}-u^{\prime}|\nabla u|^{2}\right]\right| \leq\left|u^{\prime}\right|^{-1}\left[\sqrt{a^{2}+b^{2}}\left|u_{t}^{\prime}\right|+\left|\Delta u^{\prime}\right|\right] .
$$

Therefore, using Proposition 2.4 and Cauchy's inequality, we obtain that for any $\delta \in(0,1)$,

$$
\begin{aligned}
\frac{1}{\varepsilon^{2}} \int_{Q_{2 R}} \zeta^{2} u_{3}^{2}\left|u_{x_{j}}\right|^{2} d x d t \leq & \int_{Q_{2 R}} \zeta^{2}\left|u_{x_{j}}\right|^{2}\left|u^{\prime}\right|^{-1}\left[\sqrt{a^{2}+b^{2}}\left|u_{t}^{\prime}\right|+\left|\Delta u^{\prime}\right|\right] d x d t \\
\leq & \delta \int_{Q_{2 R}} \zeta^{2}\left|u_{t}^{\prime}\right|^{2} d x d t+\delta \int_{Q_{2 R}} \zeta^{2}|\Delta u|^{2} d x d t \\
& +C_{\delta} \int_{Q_{2 R}} \zeta^{2}|\nabla u|^{4} d x d t .
\end{aligned}
$$

Substituting these estimates (with $\delta$ sufficiently small) into (3.6), and using (2.1), we can deduce that

$$
\int_{Q_{2 R}} \zeta^{2}\left|D^{2} u\right|^{2} d x d t \leq C^{\prime}\left(1+\int_{Q_{2 R}} \zeta^{2}|\nabla u|^{4} d x d t\right) .
$$

Next we estimate the term $\int_{Q_{2 R}} \zeta^{2}|\nabla u|^{4} d x d t$. By taking $\phi=\zeta|\nabla u|^{2}$ in the embedding inequality

$$
\left(\int_{G} \phi^{2} d x\right)^{1 / 2} \leq C \int_{G}(|\nabla \phi|+|\phi|) d x, \quad \forall \phi \in W^{1,1}(G),
$$


and using (2.1), we have

$$
\begin{aligned}
\int_{Q_{2 R}} \zeta^{2}|\nabla u|^{4} d x d t & \leq C\left[\int_{Q_{2 R}}\left(|\nabla \zeta||\nabla u|^{2}+2 \zeta|\nabla u|\left|D^{2} u\right|+\zeta|\nabla u|^{2}\right) d x d t\right]^{2} \\
& \leq C+C^{\prime \prime} \int_{Q_{2 R}}|\nabla u|^{2} d x d t \cdot \int_{Q_{2 R}} \zeta^{2}\left|D^{2} u\right|^{2} d x d t .
\end{aligned}
$$

Since $\sup _{t} \int_{G}|\nabla u|^{2} d x \leq C$ (cf. (2.1)), we see $C^{\prime \prime} \int_{Q_{2 R}}|\nabla u|^{2} d x d t \leq 1 /\left(4 C^{\prime}\right)$ if $R$ suitably small. Then,

$$
\int_{Q_{2 R}} \zeta^{2}|\nabla u|^{4} d x d t \leq C+\frac{1}{4 C^{\prime}} \int_{Q_{2 R}} \zeta^{2}\left|D^{2} u\right|^{2} d x d t
$$

Inserting this into 3.7$)$, we get

$$
\int_{Q_{2 R}} \zeta^{2}\left|D^{2} u\right|^{2} d x d t \leq C .
$$

Noting that $\zeta=1$ on $B_{R}\left(x_{0}\right)$, we can see the inner estimate.

Next we give the estimate near the boundary. Let $x_{0} \in \partial G$. Without loss of generality, we assume $G \cap B_{2 R}\left(x_{0}\right)=\left\{\left(x_{1}, x_{2}\right) \mid x_{2}>0\right\} \cap B_{2 R}\left(x_{0}\right)$. Choose the cut-off function $\zeta(x)$ as above. Then $(3.6)$ can be rewritten as

$$
\begin{aligned}
& \frac{a}{2} \int_{B_{2 R}} \zeta^{2}\left|u_{x_{j}}^{\prime}\right|^{2}\left(x, t_{0}+4 R^{2}\right) d x+\frac{1}{2} \int_{B_{2 R}} \zeta^{2}\left|u_{3 x_{j}}\right|^{2}\left(x, t_{0}+4 R^{2}\right) d x \\
& +\int_{Q_{2 R}} \zeta^{2}\left|u_{x_{i} x_{j}}\right|^{2} d x d t+\frac{1}{\varepsilon^{2}} \int_{Q_{2 R}} \zeta^{2}\left|u_{3 x_{j}}\right|^{2} d x d t \\
= & \frac{a}{2} \int_{B_{2 R}} \zeta^{2}\left|u_{x_{j}}^{\prime}\right|^{2}\left(x, t_{0}\right) d x+\frac{1}{2} \int_{B_{2 R}} \zeta^{2}\left|u_{3 x_{j}}\right|^{2}\left(x, t_{0}\right) d x \\
& +b \int_{Q_{2 R}} \zeta^{2} \operatorname{det}\left(u_{t}^{\prime}, u_{2 x_{j} x_{j}}^{\prime}\right) d x d t+2 b \int_{Q_{2 R}} \zeta \zeta_{x_{j}} \operatorname{det}\left(u_{t}^{\prime}, u_{x_{j}}^{\prime}\right) d x d t \\
& -2 \int_{Q_{2 R}} \zeta \zeta_{x_{j}}\left(u_{x_{j}} \cdot u_{x_{i} x_{j}}\right) d x d t+\int_{t_{0}}^{t_{0}+4 R^{2}} \int_{B_{2 R} \cap\left\{x_{2}=0\right\}} \zeta^{2}\left(u_{x_{j}} \cdot u_{x_{j} x_{2}}\right) d s d t \\
& +\int_{Q_{2 R}} \zeta^{2}|\nabla u|^{2}\left|u_{x_{j}}\right|^{2} d x d t+\frac{1}{\varepsilon^{2}} \int_{Q_{2 R}} \zeta^{2} u_{3}^{2}\left|u_{x_{j}}\right|^{2} d x d t .
\end{aligned}
$$

Except for the tenth, eleventh and twelfth terms on the right-hand side of (3.8), the others can be handled as in the argument of the inner estimate. The tenth term is

$$
\begin{aligned}
& \int_{t_{0}}^{t_{0}+4 R^{2}} \int_{B_{2 R} \cap\left\{x_{2}=0\right\}} \zeta^{2} \sum_{j=1}^{2} u_{1 x_{j}} u_{1 x_{j} x_{2}} d s d t \\
= & \int_{t_{0}}^{t_{0}+4 R^{2}} \int_{B_{2 R} \cap\left\{x_{2}=0\right\}} \zeta^{2}\left[u_{1 x_{1}} u_{1 x_{1} x_{2}}+u_{1 x_{2}}\left(\Delta u_{1}-u_{1 x_{1} x_{1}}\right)\right] d s d t .
\end{aligned}
$$


Integrating by parts and noting $\zeta=0$ at the two end points of the line segment $B_{2 R} \cap\left\{x_{2}=\right.$ $0\}$, we get

$$
\begin{aligned}
\int_{B_{2 R} \cap\left\{x_{2}=0\right\}} \zeta^{2} u_{1 x_{1}} u_{1 x_{1} x_{2}} d s & =\frac{1}{2} \int_{B_{2 R} \cap\left\{x_{2}=0\right\}} \zeta^{2}\left(u_{1 x_{1}}^{2}\right)_{x_{2}} d s \\
& =-\frac{1}{2} \int_{B_{2 R} \cap\left\{x_{2}=0\right\}}\left(\zeta^{2}\right)_{x_{2}} u_{1 x_{1}}^{2} d s
\end{aligned}
$$

Since (2.2) implies $\Delta u_{1}=a u_{1 t}-b u_{2 t}-u_{1}|\nabla u|^{2}-\frac{1}{\varepsilon^{2}} u_{1} u_{3}^{2}=-u_{1}|\nabla u|^{2}$ on $\partial G$, we have

$$
\begin{aligned}
\int_{B_{2 R} \cap\left\{x_{2}=0\right\}} \zeta^{2} u_{1 x_{2}} \Delta u_{1} d s & =-\int_{B_{2 R} \cap\left\{x_{2}=0\right\}} \zeta^{2} u_{1 x_{2}} u_{1}|\nabla u|^{2} d s \\
& =-\frac{1}{2} \int_{B_{2 R} \cap\left\{x_{2}=0\right\}} \zeta^{2}\left(u_{1}^{2}\right)_{x_{2}}|\nabla u|^{2} d s .
\end{aligned}
$$

Similarly, we have the same results for the eleventh and twelfth terms. Noting $|u|=1$, and using (3.1) and Cauchy's inequality, we know that these terms are

$$
\begin{aligned}
& -\int_{t_{0}}^{t_{0}+4 R^{2}} \int_{B_{2 R} \cap\left\{x_{2}=0\right\}}\left[\frac{1}{2}\left(\zeta^{2}\right)_{x_{2}}\left(u_{x_{1}}\right)^{2}+\frac{1}{2} \zeta^{2}\left(|u|^{2}\right)_{x_{2}}|\nabla u|^{2}+\zeta^{2}\left(u_{x_{2}} \cdot u_{x_{1} x_{1}}\right)\right] d s d \tau \\
\leq & -\frac{1}{2} \int_{t_{0}}^{t_{0}+4 R^{2}} \int_{B_{2 R} \cap\left\{x_{2}=0\right\}}\left(\zeta^{2}\right)_{x_{2}}\left(g_{x_{1}}\right)^{2} d s d \tau \\
& +C \int_{t_{0}}^{t_{0}+4 R^{2}} \int_{B_{2 R} \cap\left\{x_{2}=0\right\}} \zeta^{2}\left[\left(u_{x_{2}}\right)^{2}+\left(g_{x_{1} x_{1}}\right)^{2}\right] d s d \tau \leq C .
\end{aligned}
$$

Inserting this result into (3.8), we can also deduce the estimate of the second-order terms near the boundary. Thus, $(3.2)$ is proved and hence Proposition 3.2 is complete.

Finally, we gave a uniform $W_{p}^{2,1}$-estimate for some $p>2$. Although it seems difficult to do for $u_{\varepsilon}$, we can handle $u_{\varepsilon 3}$ since the second equation of 1.1 is parabolic.

Proposition 3.3. Assume that $u_{\varepsilon}: G_{T} \rightarrow S^{2}$ is a solution to $\overline{1.1}$. Let $\left(x_{0}, t_{0}\right) \in \overline{G_{T}}$, $Q_{R, r}=\overline{G_{T}} \cap\left(B_{R}\left(x_{0}\right) \times\left[t_{0}, t_{0}+r\right]\right)$. Then we can find $p>2$ and $C>0$ (which is independent of $\varepsilon$ ) such that

$$
\left\|u_{\varepsilon 3}\right\|_{W_{p}^{2,1}\left(Q_{R / 2, r / 2}\right)} \leq C .
$$

Proof. The second equation in $(1.1)$ is

$$
u_{3 t}=\Delta u_{3}+u_{3}|\nabla u|^{2}-\frac{1}{\varepsilon^{2}} u_{3}\left|u^{\prime}\right|^{2} .
$$

Set $\psi=\frac{1}{\varepsilon^{2}} u_{3}(x, t)$. Then

$$
\psi\left|u^{\prime}\right|^{2}+\varepsilon^{2} \psi_{t}=\varepsilon^{2} \Delta \psi+\varepsilon^{2} \psi|\nabla u|^{2} .
$$


Let the cut-off function $\zeta \in C_{0}^{\infty}\left(Q_{R, r}\right)$ satisfy $\zeta=1$ on $Q_{R / 2, r / 2}$. Multiplying (3.11) with $\psi^{p-1} \zeta^{p}(p>2)$ and integrating on $Q_{R, r}$, we get

$$
\begin{aligned}
& \int_{Q_{R, r}} \psi^{p} \zeta^{p}\left|u^{\prime}\right|^{2} d x d t+\varepsilon^{2} \int_{Q_{R, r}} \psi_{t} \psi^{p-1} \zeta^{p} d x d t \\
= & \varepsilon^{2} \int_{Q_{R, r}}(\Delta \psi) \psi^{p-1} \zeta^{p} d x d t+\varepsilon^{2} \int_{Q_{R, r}} \psi^{p} \zeta^{p}|\nabla u|^{2} d x d t .
\end{aligned}
$$

Integrating by parts and noting $\psi=0$ on $\partial G_{T}$, we have

$$
\varepsilon^{2} \int_{Q_{R, r}} \psi_{t} \psi^{p-1} \zeta^{p} d x d t=-\varepsilon^{2} \int_{Q_{R, r}} \psi^{p} \zeta^{p-1} \zeta_{t} d x d t
$$

and

$$
\begin{aligned}
\varepsilon^{2} \int_{Q_{R, r}}(\Delta \psi) \psi^{p-1} \zeta^{p} d x d t= & -\varepsilon^{2} \int_{Q_{R, r}} \nabla \psi \nabla\left(\psi^{p-1} \zeta^{p}\right) d x d t \\
= & -\varepsilon^{2}(p-1) \int_{Q_{R, r}} \psi^{p-2} \zeta^{p}|\nabla \psi|^{2} d x d t \\
& -\varepsilon^{2} p \int_{Q_{R, r}} \psi^{p-1} \zeta^{p-1} \nabla \psi \nabla \zeta d x d t .
\end{aligned}
$$

Inserting (3.13) and (3.14) into (3.12), and applying Young's inequality, we can obtain that for any $\delta \in(0,1)$,

$$
\begin{aligned}
& \int_{Q_{R, r}} \psi^{p} \zeta^{p}\left|u^{\prime}\right|^{2} d x d t+\varepsilon^{2}(p-1) \int_{Q_{R, r}} \psi^{p-2} \zeta^{p}|\nabla \psi|^{2} d x d t \\
= & \varepsilon^{2} \int_{Q_{R, r}}\left[\psi^{p} \zeta^{p-1} \zeta_{t}-p \psi^{p-1} \zeta^{p-1} \nabla \psi \nabla \zeta+\psi^{p} \zeta^{p}|\nabla u|^{2}\right] d x d t \\
\leq & \delta \int_{Q_{R, r}} \psi^{p} \zeta^{p} d x d t+C(\delta) \int_{Q_{R, r}} \varepsilon^{2 p}\left(|\psi|^{p}\left|\zeta_{t}\right|^{p}+p^{p}|\nabla \psi|^{p}|\nabla \zeta|^{p}+|\psi|^{p} \zeta^{p}|\nabla u|^{2 p}\right) d x d t .
\end{aligned}
$$

Obviously, $\varepsilon^{2} \psi=u_{3}, \varepsilon^{2} \nabla \psi=\nabla u_{3}$. Noting Proposition 2.4 and choosing $\delta$ sufficiently small, we get

$$
\int_{Q_{R, r}} \psi^{p} \zeta^{p} d x d t \leq C \int_{Q_{R, r}}\left(\left|u_{3}\right|^{p}\left|\zeta_{t}\right|^{p}+\left|\nabla u_{3}\right|^{p}|\nabla \zeta|^{p}+\left|u_{3}\right|^{p} \zeta^{p}|\nabla u|^{2 p}\right) d x d t .
$$

Using 2.1) and (3.2), we have $\|u\|_{W_{2}^{2,1}\left(Q_{R, r}\right)} \leq C$. This implies that

$$
\|u\|_{L^{2}\left(t_{0}, t_{0}+r^{2} ; H^{2}\left(G \cap B_{R}\right)\right)}+\left\|u_{t}\right\|_{L^{2}\left(t_{0}, t_{0}+r^{2} ; L^{2}\left(G \cap B_{R}\right)\right)} \leq C .
$$

Clearly, for all $q>4, H^{2}\left(G \cap B_{R}\right) \subset W^{1, q}\left(G \cap B_{R}\right) \subset L^{2}\left(G \cap B_{R}\right)$ and the imbedding map from $H^{2}\left(G \cap B_{R}\right)$ to $L^{2}\left(G \cap B_{R}\right)$ is compact. According to Corollary 4 in 19, by (3.16) we obtain that

$$
\|u\|_{C\left(t_{0}, t_{0}+r^{2} ; W^{1, q}\left(G \cap B_{R}\right)\right)} \leq C \text { for all } q>4 .
$$


Here $C>0$ is independent of $\varepsilon$. This implies

$$
\|\nabla u\|_{L^{q}\left(Q_{R, r}\right)} \leq C \text { for all } q>4
$$

Noting $\zeta=1$ on $Q_{R / 2, r / 2}$, and using (3.15) and (3.17), we obtain that for some $p>2$, there exists a positive constant $C$ which is independent of $\varepsilon$,

$$
\int_{Q_{R / 2, r / 2}} \psi^{p} d x d t \leq C
$$

Set $F_{\varepsilon}(x, t)=\left[u_{3}|\nabla u|^{2}-\frac{1}{\varepsilon^{2}} u_{3}\left|u^{\prime}\right|^{2}\right](x, t)$. Then $(3.10)$ becomes

$$
u_{3 t}=\Delta u_{3}+F_{\varepsilon} .
$$

Using (3.17) and (3.18), we get $\left\|F_{\varepsilon}\right\|_{L^{p}\left(G_{T}\right)} \leq C$ for some $p>2$, where $C$ is independent of $\varepsilon$. Therefore, applying $L^{p}$ theory for the parabolic equation (3.19), we know that (3.9) is true. Proposition 3.3 is proved.

Proof of Theorem 1.1. According to Proposition 2.2, the first three results of Theorem 1.1 are proved.

By Proposition 3.3 and the $t$-anisotropy embedding inequality, we have

$$
\left|u_{\varepsilon 3}\right|_{\gamma, \gamma / 2 ; \overline{G_{T}}} \leq C, \quad 0<\gamma<\min \{1,2-4 / p\}
$$

where $C>0$ is independent of $\varepsilon$. This and Proposition 2.2 imply that there exists a subsequence $u_{\varepsilon_{k} 3}$ of $u_{\varepsilon 3}$ such that

$$
u_{\varepsilon_{k} 3} \rightarrow 0 \quad \text { in } C^{\alpha, \alpha / 2}\left(\overline{G_{T}}\right)
$$

for all $\alpha \in(0, \gamma)$ when $\varepsilon_{k} \rightarrow 0$. Since all the subsequences converge to the same limit 0 , the convergence above still hold for $u_{\varepsilon 3}$. Theorem 1.1 is complete.

Remark 3.4. Noting $\left|u_{\varepsilon}^{\prime}\right|=\sqrt{1-u_{\varepsilon 3}^{2}}$, we get

$$
\left|u_{\varepsilon}^{\prime}\right| \rightarrow 1 \quad \text { in } C^{\alpha, \alpha / 2}\left(\overline{G_{T}}\right) .
$$

\section{Non-parabolic behavior}

Scale the solution $u_{\varepsilon}$ to 1.1 in time

$$
v_{\varepsilon}(x, t)=u_{\varepsilon}(x, \varepsilon t) .
$$


Then $v_{\varepsilon}=\left(v_{\varepsilon}^{\prime}, v_{\varepsilon 3}\right)$ satisfies

$$
\begin{cases}(a+i b) \frac{1}{\varepsilon} v_{t}^{\prime}=\Delta v^{\prime}+v^{\prime}|\nabla v|^{2}+\frac{1}{\varepsilon^{2}} v^{\prime} v_{3}^{2} & \text { on } G_{T}, \\ \frac{1}{\varepsilon} v_{3 t}=\Delta v_{3}+v_{3}|\nabla v|^{2}+\frac{1}{\varepsilon^{2}} v_{3}\left(v_{3}^{2}-1\right) & \text { on } G_{T}, \\ \left.v\right|_{\partial G \times \mathbb{R}^{+}}=g(x), & \\ v(x, 0)=u_{0}(x), & x \in G .\end{cases}
$$

Similar to Proposition 2.1, we also have a conservation result.

Proposition 4.1. Assume $v_{\varepsilon}: G \times \mathbb{R}^{+} \rightarrow S^{2}$ satisfies (4.1). Then

$$
\sup _{t>0}\left[\int_{0}^{t} \int_{G}\left(\frac{a}{\varepsilon}\left|\frac{\partial}{\partial t} v_{\varepsilon}^{\prime}\right|^{2}+\frac{1}{\varepsilon}\left|\frac{\partial}{\partial t} v_{\varepsilon 3}\right|^{2}\right) d x d \tau+E_{\varepsilon}\left(v_{\varepsilon}(x, t)\right)\right]=\frac{1}{2}\left\|\nabla u_{0}\right\|_{2}^{2} .
$$

Here

$$
E_{\varepsilon}(v)=\frac{1}{2} \int_{G}|\nabla v|^{2} d x+\frac{1}{2 \varepsilon^{2}} \int_{G}\left|v_{3}\right|^{2} d x .
$$

Proof. Similar to the derivation of $2.2-2.4$, we have the following three equations

$$
\begin{aligned}
& \frac{a}{\varepsilon} v_{1 t}-\frac{b}{\varepsilon} v_{2 t}=\Delta v_{1}+v_{1}|\nabla v|^{2}+\frac{1}{\varepsilon^{2}} v_{1} v_{3}^{2}, \\
& \frac{b}{\varepsilon} v_{1 t}+\frac{a}{\varepsilon} v_{2 t}=\Delta v_{2}+v_{2}|\nabla v|^{2}+\frac{1}{\varepsilon^{2}} v_{2} v_{3}^{2}, \\
& \frac{1}{\varepsilon} v_{3 t}=\Delta v_{3}+v_{3}|\nabla v|^{2}+\frac{1}{\varepsilon^{2}} v_{3}\left(v_{3}^{2}-1\right) .
\end{aligned}
$$

Multiply (4.3), 4.4 and 4.5) with $v_{1 t}, v_{2 t}$ and $v_{3 t}$, respectively. Integrating over $G$, we have

$$
\begin{aligned}
& \frac{a}{\varepsilon} \int_{G}\left|v_{1 t}\right|^{2} d x-\frac{b}{\varepsilon} \int_{G} v_{1 t} v_{2 t} d x \\
&= \int_{G} v_{1 t} \Delta v_{1} d x+\frac{1}{2} \int_{G}\left(\left|v_{1}\right|^{2}\right)_{t}|\nabla v|^{2} d x+\frac{1}{2 \varepsilon^{2}} \int_{G}\left(\left|v_{1}\right|^{2}\right)_{t} v_{3}^{2} d x \\
& \frac{b}{\varepsilon} \int_{G} v_{1 t} v_{2 t} d x+\frac{a}{\varepsilon} \int_{G}\left|v_{2 t}\right|^{2} d x \\
&= \int_{G} v_{2 t} \Delta v_{2} d x+\frac{1}{2} \int_{G}\left(\left|v_{2}\right|^{2}\right)_{t}|\nabla v|^{2} d x+\frac{1}{2 \varepsilon^{2}} \int_{G}\left(\left|v_{2}\right|^{2}\right)_{t} v_{3}^{2} d x \\
& \frac{1}{\varepsilon} \int_{G}\left|v_{3 t}\right|^{2} d x=\int_{G} v_{3 t} \Delta v_{3} d x+\frac{1}{2} \int_{G}\left(\left|v_{3}\right|^{2}\right)_{t}|\nabla v|^{2} d x+\frac{1}{2 \varepsilon^{2}} \int_{G}\left(\left|v_{3}\right|^{2}\right)_{t}\left(v_{3}^{2}-1\right) d x
\end{aligned}
$$

Noting $v_{t}=0$ on $\partial G$, we obtain by Green's theorem that

$$
\int_{G} v_{i t} \Delta v_{i} d x=-\frac{d}{2 d t} \int_{G}\left|\nabla v_{i}\right|^{2} d x, \quad i=1,2,3 .
$$

Inserting these into (4.6), (4.7) and (4.8), respectively. Noting $|v|^{2}=1$, we have

$$
\int_{G} \frac{a}{\varepsilon}\left(\left|v_{1 t}\right|^{2}+\left|v_{2 t}\right|^{2}\right) d x+\int_{G} \frac{1}{\varepsilon}\left|v_{3 t}\right|^{2} d x=-\frac{d}{d t} E_{\varepsilon}(v(x, t)) .
$$


Integrating from 0 to $t$, we deduce that, for all $t>0$,

$$
\int_{0}^{t} \int_{G}\left(\frac{a}{\varepsilon}\left|\frac{\partial}{\partial t} v_{\varepsilon}^{\prime}\right|^{2}+\frac{1}{\varepsilon}\left|\frac{\partial}{\partial t} v_{\varepsilon 3}\right|^{2}\right) d x d \tau+E_{\varepsilon}\left(v_{\varepsilon}(x, t)\right)=E_{\varepsilon}(v(x, 0))=\frac{1}{2}\left\|\nabla u_{0}\right\|_{2}^{2} .
$$

Proposition 4.1 is proved.

Proof of Theorem 1.2. By Proposition 4.1, we can find a positive constant $C$ (independent of $\varepsilon$ ) such that

$$
\left\|v_{\varepsilon}\right\|_{L^{\infty}\left(0, T ; H^{1}\left(G, S^{2}\right)\right)} \leq C \quad \text { and } \quad\left\|\frac{\partial v_{\varepsilon}}{\partial t}\right\|_{L^{2}\left(0, T ; L^{2}\left(G, S^{2}\right)\right)} \leq C .
$$

Hence, there is a subsequence $\varepsilon_{k}$ such that as $\varepsilon_{k} \rightarrow 0$,

$$
\begin{aligned}
v_{\varepsilon_{k}} & \rightarrow h & & \text { weakly* in } L^{\infty}\left(0, T ; H^{1}\left(G, S^{2}\right)\right), \\
\frac{\partial}{\partial t} v_{\varepsilon_{k}} & \rightarrow \frac{\partial}{\partial t} h & & \text { weakly in } L^{2}\left(0, T ; L^{2}\left(G, S^{2}\right)\right), \\
v_{\varepsilon_{k}} & \rightarrow h & & \text { in } L^{2}\left(0, T ; L^{2}\left(G, S^{2}\right)\right) .
\end{aligned}
$$

Next, we prove that $h=\left(v_{*}^{\prime}, 0\right)$ satisfies the conditions in Theorem 1.2 . When $\varepsilon \rightarrow 0$, by 4.2 we get $\sup _{t>0} \int_{G} v_{3}^{2} d x \rightarrow 0$. This result shows

$$
h_{3}=0 \quad \text { a.e. on } G_{T} \text {. }
$$

Since $v_{\varepsilon_{k}}=v=\left(v^{\prime}, v_{3}\right)$ satisfies (4.1), multiply the first equation in (4.1) by $\bar{v}^{\prime}$. Taking the real part and imaginary part, we get

$$
\begin{gathered}
\frac{a}{2}\left(\frac{\left|v^{\prime}\right|^{2}}{\varepsilon}\right)_{t}+\frac{b}{\varepsilon}\left(v_{1 t} v_{2}-v_{2 t} v_{1}\right)=v_{1} \Delta v_{1}+v_{2} \Delta v_{2}+\left|v^{\prime}\right|^{2}|\nabla v|^{2}+\frac{1}{\varepsilon^{2}}\left|v^{\prime}\right|^{2} v_{3}^{2} \\
\frac{b}{2}\left(\frac{\left|v^{\prime}\right|^{2}}{\varepsilon}\right)_{t}+\frac{a}{\varepsilon}\left(v_{2 t} v_{1}-v_{1 t} v_{2}\right)=v_{1} \Delta v_{2}-v_{2} \Delta v_{1}
\end{gathered}
$$

Multiplying the second equation in (4.1) by $v_{3}$, we have

$$
\frac{1}{2}\left(\frac{v_{3}^{2}}{\varepsilon}\right)_{t}=v_{3} \Delta v_{3}+v_{3}^{2}|\nabla v|^{2}-\frac{1}{\varepsilon^{2}}\left|v^{\prime}\right|^{2} v_{3}^{2} .
$$

Calculating by $a \times 4.9+b \times(4.10)+a \times 4.11$, we obtain

$$
\frac{a-a^{2}-b^{2}}{2}\left(\frac{v_{3}^{2}}{\varepsilon}\right)_{t}=a\left(v_{1} \Delta v_{1}+v_{2} \Delta v_{2}+v_{3} \Delta v_{3}\right)+b\left(v_{1} \Delta v_{2}-v_{2} \Delta v_{1}\right)+a|\nabla v|^{2} .
$$

In view of $a \neq a^{2}+b^{2}$, it follows that

$$
\left(\frac{v_{3}^{2}}{\varepsilon}\right)_{t}=\frac{2}{a-a^{2}-b^{2}}\left[a \operatorname{Re}\left(\bar{v}^{\prime} \Delta v^{\prime}\right)+a v_{3} \Delta v_{3}+b \operatorname{Im}\left(\bar{v}^{\prime} \Delta v^{\prime}\right)+a|\nabla v|^{2}\right] .
$$


Integrating from 0 to $t$, we get

$$
\frac{v_{3}^{2}}{\varepsilon}=\frac{2}{a-a^{2}-b^{2}} \int_{0}^{t}\left[a \operatorname{Re}\left(\bar{v}^{\prime} \Delta v^{\prime}\right)+a v_{3} \Delta v_{3}+b \operatorname{Im}\left(\bar{v}^{\prime} \Delta v^{\prime}\right)+a|\nabla v|^{2}\right] d \tau .
$$

By Proposition 4.1, $v_{3}^{2} / \varepsilon \rightarrow w$ in $\mathcal{D}^{\prime}$ for some nonnegative function $w$. By 4.12 , we know that

$$
w=\frac{2}{a-a^{2}-b^{2}} \int_{0}^{t}\left[a \operatorname{Re}\left(\bar{v}_{*}^{\prime} \Delta v_{*}^{\prime}\right)+b \operatorname{Im}\left(\bar{v}_{*}^{\prime} \Delta v_{*}^{\prime}\right)+a\left|\nabla v_{*}\right|^{2}\right] d \tau .
$$

Multiplying the first equation in (4.1) by $\varepsilon$ and letting $\varepsilon \rightarrow 0$, we get

$$
(a+i b) v_{* t}^{\prime}=\frac{2 v_{*}^{\prime}}{a-a^{2}-b^{2}} \int_{0}^{t}\left[a \operatorname{Re}\left(\bar{v}_{*}^{\prime} \Delta v_{*}^{\prime}\right)+b \operatorname{Im}\left(\bar{v}_{*}^{\prime} \Delta v_{*}^{\prime}\right)+a\left|\nabla v_{*}\right|^{2}\right] d \tau .
$$

Moreover, if we write $v_{*}=\left(e^{i \theta}, 0\right)$, the result above implies

$$
(a i-b) \theta_{t}=\frac{2 b}{a-a^{2}-b^{2}} \int_{0}^{t} \Delta \theta d \tau \text {. }
$$

So we have the following non-parabolic results

$$
a \theta_{t}=0, \quad b \theta_{t}=\frac{2 b}{a^{2}+b^{2}-a} \int_{0}^{t} \Delta \theta d \tau .
$$

If $a>0$, then $\theta_{t}=0$, and we know $\theta$ only depends on $x$. Moveover, when $b \neq 0$, we have $\Delta \theta=0$, i.e., $v_{*}^{\prime}$ is a harmonic map. If $a=0$, then $b \neq 0$ and hence

$$
\theta_{t t}=\frac{2}{b^{2}} \Delta \theta
$$

which implies that $\left(v_{*}^{\prime}, 0\right)$ satisfies 1.5 .

Since the limit $\left(v_{*}^{\prime}, 0\right)$ is unique, the convergence above can be generalized to all $\varepsilon$ instead of the subsequence $\varepsilon_{k}$. Theorem 1.2 is proved.

\section{Acknowledgments}

The authors would like to thank the referees for their valuable comments. He is also grateful to Qian Zhang for many fruitful discussions. This research was supported by NNSF (11871278) of China and NSF of Jiangsu Education Commission (19KJB110016).

\section{References}

[1] F. Alouges and A. Soyeur, On global weak solutions for Landau-Lifshitz equations: existence and nonuniqueness, Nonlinear Anal. 18 (1992), no. 11, 1071-1084.

[2] F. Bethuel, G. Orlandi and D. Smets, Convergence of the parabolic Ginzburg-Landau equation to motion by mean curvature, Ann. of Math. (2) 163 (2006), no. 1, 37-163. 
[3] T. Colin and A. Soyeur, Some singular limits for evolutionary Ginzburg-Landau equations, Asymptotic Anal. 13 (1996), no. 4, 361-372.

[4] A. de Laire, Minimal energy for the traveling waves of the Landau-Lifshitz equation, SIAM J. Math. Anal. 46 (2014), no. 1, 96-132.

[5] A. de Laire and P. Gravejat, The Sine-Gordon regime of the Landau-Lifshitz equation with a strong easy-plane anisotropy, Ann. Inst. H. Poincaré Anal. Non Linéaire 35 (2018), no. 7, 1885-1945.

[6] S. Ding and Z. Liu, Hölder convergence of Ginzburg-Landau approximations to the harmonic map heat flow, Nonlinear Anal. 46 (2001), no. 6, Ser. A: Theory Methods, $807-816$.

[7] X. Fu, A weighted identity for partial differential operators of second order and its applications, C. R. Math. Acad. Sci. Paris 342 (2006), no. 8, 579-584.

[8] B. Guo and S. Ding, Landau-Lifshitz Equations, Frontiers of Research with the Chinese Academy of Sciences 1, World Scientific, Hackensack, NJ, 2008.

[9] R. L. Jerrard and D. Smets, Leapfrogging vortex rings for the three dimensional GrossPitaevskii equation, Ann. PDE 4 (2018), no. 1, Art. 4, 48 pp.

[10] S. Komineas and N. Papanicolaou, Vortex dynamics in two-dimensional antiferromagnets, Nonlinearity 11 (1998), no. 2, 265-290.

[11] Y. Lei, A uniqueness result on the solution for a Landau-Lifshitz system with penalization, Nonlinear Anal. 69 (2008), no. 2, 770-780.

[12] E. H. Lieb and M. Loss, Analysis, Second edition, Graduate Studies in Mathematics 14, American Mathematical Society, Providence, RI, 2001.

[13] F. H. Lin, Some dynamical properties of Ginzburg-Landau vortices, Comm. Pure Appl. Math. 49 (1996), no. 4, 323-359.

[14] F.-H. Lin and J. X. Xin, On the incompressible fluid limit and the vortex motion law of the nonlinear Schrödinger equation, Comm. Math. Phys. 200 (1999), no. 2, $249-274$.

[15] R. Moser, Partial Regularity for Harmonic Maps and Related Problems, World Scientific, Hackensack, NJ, 2005.

[16] N. Papanicolaou and P. N. Spathis, Semitopological solitons in planar ferromagnets, Nonlinearity 12 (1999), no. 2, 285-302. 
[17] L. Rosier and B.-Y. Zhang, Null controllability of the complex Ginzburg-Landau equation, Ann. Inst. H. Poincaré Anal. Non Linéaire 26 (2009), no. 2, 649-673.

[18] S. Serfaty, Mean field limits of the Gross-Pitaevskii and parabolic Ginzburg-Landau equations, J. Amer. Math. Soc. 30 (2017), no. 3, 713-768.

[19] J. Simon, Compact Sets in the Space $L^{p}(0, T ; B)$, Ann. Math. Pura Appl. (4) 146 (1987), 65-96.

[20] R. Venkatraman, Periodic orbits of Gross-Pitaevskii in the disc with vortices following point vortex flow, Calc. Var. Partial Differential Equations 56 (2017), no. 3, Art. 64, $35 \mathrm{pp}$.

Yutian Lei

Jiangsu Key Laboratory for NSLSCS, School of Mathematical Sciences, Nanjing Normal University, Nanjing, 210023, China

E-mail address: leiyutian@njnu.edu.cn 